This Section of Epidemiology and Psychiatric Sciences appears in each issue of the Journal to stress the role of the epidemiological approach to promote advances in the field of clinical psychopharmacology, with a particular attention to controversial findings. The ultimate aims are to help develop a more critical attitude towards the results of research studies published in the international literature, to promote original research projects with higher methodological standards, and to implement the most relevant results of research in every-day clinical practice. These contributions are written in house by the journal's editorial team or commissioned by the Section Editor (no more than 1000 words, short unstructured abstract, 4 key-words, one Table or Figure and up to ten references).

Corrado Barbui, Section Editor

\title{
What does the latest meta-analysis really tell us about antidepressants?
}

\author{
J. Moncrieff* \\ Division of Psychiatry, University College London, London, UK
}

A recent meta-analysis of antidepressant trials is the largest conducted to date. Although it claims to prove antidepressant effectiveness beyond dispute, the main outcome is response rates, which are derived from continuous data in a process that can inflate differences between groups. The standardised mean difference of 0.3 is in line with other meta-analyses that show small differences between antidepressants and placebo that are unlikely to be clinically significant. Other factors likely to exaggerate the effects are discussed, and evidence on associations between antidepressant effects and severity and outcomes of long-term treatment is considered. Clinicians need to have open discussions with patients about the limitations of antidepressant research, the lack of evidence that antidepressants correct a chemical imbalance or other brain abnormality, and the range of adverse effects and mental and physical alterations they can produce.

Received 20 April 2018; Accepted 28 April 2018; First published online 28 May 2018

Key words: Antidepressant efficacy, antidepressants, meta-analysis.

A recent meta-analysis of antidepressant trials (Cipriani et al. 2018) was greeted by media coverage declaring that 'the drugs do work' and that doubts about their usefulness should be 'put to rest'. Some of the authors called for wider prescribing, suggesting that one million more people in the UK should be prescribed antidepressants (Boseley, 2018).

The latest meta-analysis, undertaken by Cipriani et al., is the largest to have been undertaken, and included some unpublished as well as published studies. It employed a network analysis method, including data from comparative trials that did not include a placebo group. Included trials evaluated short-term treatment with antidepressants for an average of 8 weeks.

\footnotetext{
* Address for correspondence: J. Moncrieff, Division of Psychiatry, University College London, London, UK.

(Email: j.moncrieff@ucl.ac.uk)
}

The principal efficacy outcome, 'response' rate, showed odds ratios of between 2.13 for amitriptyline and 1.37 for reboxetine, indicating that patients treated with antidepressants were approximately one and a half to two times more likely to show a response than patients treated with a placebo (Cipriani et al. 2018).

'Response' is an artificial category, however. It is arbitrarily constructed out of scores on depression rating scales, such as the Hamilton Rating Scale for Depression (HRSD), which is the data that are actually collected from participants. The assumption that a 50\% decrease in scores (the usual definition of response) represents a clinically meaningful improvement has not been validated. Moreover, categorising data can inflate small differences in raw scores, especially if the cut-off is close to the mean score, which it frequently is (Kirsch \& Moncrieff, 2007).

The standardised mean difference (SMD) for the difference in rating scale scores in the Cipriani et al. (2018) 
meta-analysis was 0.3 , which is comparable with several other meta-analyses conducted over the last two decades. These show that SMDs of around 0.3 translate into differences in HRSD scores of around two points (Kirsch et al. 2002), which is unlikely to be clinically relevant. Research comparing HRSD scores with scores on the Clinical Global Impressions (CGI) scale suggest that such a difference would not even be noticed, with a difference of at least eight points being required to register as 'mild improvement' (Moncrieff \& Kirsch, 2015).

However, even these small differences are easily accounted for by the fact that antidepressants produce more or less subtle mental and physical alterations (e. g. nausea, dry mouth, dizziness, drowsiness and emotional blunting) irrespective of whether or not they treat depression. These alterations enable participants to guess whether they have been allocated to antidepressant or placebo better than would be expected by chance. Participants receiving the active drugs may therefore experience amplified placebo effects. This may explain why antidepressants that cause the most noticeable alterations, such as amitriptyline, appeared to be the most effective in the Cipriani et al. (2018) meta-analysis.

Another problem is that antidepressant trials often include people who are already on antidepressants, and who are withdrawn from them prior to the trial. Although there is usually a 'placebo washout' period during which participants are withdrawn from previous medication, we know that withdrawal effects can persist for longer than the 1-2 weeks that such periods usually last. Since trials do not try to identify antidepressant withdrawal effects, they may be misclassified as symptoms of depression in people who are subsequently randomised to placebo.

The use of network meta-analysis is also likely to have inflated differences between antidepressants and placebo. People treated with antidepressants in placebo-controlled trials show lower response rates than participants in comparative trials that lack a placebo group, indicating how outcomes are influenced by expectations of receiving active therapy (Sinyor et al. 2010). Therefore, network analysis includes data on antidepressants that are collected in more favourable circumstances than the data on placebo.

The Cipriani et al. meta-analysis only looked at data on short-term treatment, whereas in real-life people increasingly take antidepressants for years. Few randomised, placebo-controlled trials have investigated longterm effects. In the large longitudinal STAR-D study, the proportion of people who adhered to recommended treatment, recovered and did not relapse was startlingly low (only 108 out of the 3110 people who satisfied inclusion criteria) (Pigott et al. 2010).
Some evidence indicates that people with more severe depression show greater relative differences between antidepressants and placebo than those with less severe symptoms. However, other studies have not detected an association with severity (Walsh et al. 2002; Gibbons et al. 2012). In one meta-analysis that detected a severity effect, the difference between drug and placebo among those with severe depression was four points on the HRSD, which still falls well below the level that would indicate minimal improvement on the CGI (Kirsch et al. 2008).

The recent meta-analysis does not resolve recent debates about the utility of antidepressants, but clinical guidelines continue to recommend them, and many people expect to be offered them. Much of the general public has also been persuaded that depression is caused by a chemical 'imbalance' that antidepressants correct, even though the evidence for this idea was never convincing. Indeed, despite speculation about a number of physiological processes, the mechanism through which antidepressants might affect symptoms of depression remains unknown.

Antidepressants are not placebos, however. They are active drugs that produce a range of physical and mental alterations. Some of the alterations they produce, such as the sedation induced by tricyclics, or the emotional blunting associated with selective serotonin reuptake inhibitors, may plausibly be useful for various psychological symptoms. However, the evidence reviewed above suggests the utility of these effects for the treatment of depression specifically is minimal. Moreover, some alterations are harmful or unpleasant, such as sexual dysfunction, which in some cases seems to persist after discontinuation of the drug (Farnsworth \& Dinsmore, 2009) and agitation, suicidal and aggressive behaviour which can occur among younger users in particular (Sharma et al. 2016). Although serious adverse effects are probably rare, we have little data on the frequency or mechanisms of such effects, or how to treat them.

Patients and clinicians need to consider all these factors if they are to make informed decisions about whether to use antidepressants or not. In particular, clinicians need to discuss the limitations of research on the efficacy and effectiveness of antidepressants, the lack of support for the idea that antidepressants correct a chemical imbalance or other brain abnormality and the range of adverse effects and mental and physical alterations that antidepressants can produce.

\section{Financial Support}

The research received no specific grant form any funding agency, commercial or not for profit sectors. 


\section{Conflict of Interest}

The author has no financial conflict of interests. She is co-chair person of the Critical Psychiatry Network, an international group of psychiatrists who challenge the dominance of the biomedical approach in psychiatry.

\section{Ethical Standard}

The authors assert that all procedures relating to this work comply with the standards of the relevant national and international committees on human experimentation and with the Helsinki Declaration of 1975 as revised in 2008 .

\section{References}

Boseley S (2018). The drugs do work: antidepressants are effective study shows. The Guardian, 21-2-2018.

Cipriani A, Furukawa TA, Salanti G, Chaimani A, Atkinson LZ, Ogawa Y, Leucht S, Ruhe HG, Turner EH, Higgins JPT, Egger M, Takeshima N, Hayasaka Y, Imai H, Shinohara K, Tajika A, Ioannidis JPA, Geddes JR (2018). Comparative efficacy and acceptability of 21 antidepressant drugs for the acute treatment of adults with major depressive disorder: a systematic review and network meta-analysis. The Lancet 391, 1357-1366.

Farnsworth KD, Dinsmore WW (2009). Persistent sexual dysfunction in genitourinary medicine clinic attendees induced by selective serotonin reuptake inhibitors. International Journal of STD and AIDS 20, 68-69.

Gibbons RD, Hur K, Brown CH, Davis JM, Mann JJ (2012). Benefits from antidepressants: synthesis of 6-week patient-level outcomes from double-blind placebo-controlled randomized trials of fluoxetine and venlafaxine. Archives General Psychiatry 69, 572-579.

Kirsch I, Moncrieff J (2007). Clinical trials and the response rate illusion. Contemporary Clinical Trials 28, 348-351.

Kirsch I, Moore TJ, Scoboria A, Nicholls SS (2002). The emperor's new drugs: an analysis of antidepressant medication data submitted to the US Food and Drug Administration. Prevention and Treatment 5, 23.

Kirsch I, Deacon BJ, Huedo-Medina TB, Scoboria A, Moore TJ, Johnson BT (2008). Initial severity and antidepressant benefits: a meta-analysis of data submitted to the Food and Drug Administration. PLoS Medicine 5, e45.

Moncrieff J, Kirsch I (2015). Empirically derived criteria cast doubt on the clinical significance of antidepressant-placebo differences. Contempemporary Clinical Trials 43, 60-62.

Pigott HE, Leventhal AM, Alter GS, Boren JJ (2010). Efficacy and effectiveness of antidepressants: current status of research. Psychotherapy and Psychosomatics 79, 267-279.

Sharma T, Guski LS, Freund N, Gotzsche PC (2016). Suicidality and aggression during antidepressant treatment: systematic review and meta-analyses based on clinical study reports. Brisith Medical Journal 352, i65.

Sinyor M, Levitt AJ, Cheung AH, Schaffer A, Kiss A, Dowlati Y, Lanctot KL (2010). Does inclusion of a placebo arm influence response to active antidepressant treatment in randomized controlled trials? Results from pooled and meta-analyses. Journal of Clinical Psychiatry 71, 270-279.

Walsh BT, Seidman SN, Sysko R, Gould M (2002). Placebo response in studies of major depression: variable, substantial, and growing. Journal of the American Medical Association 287, 1840-1847. 